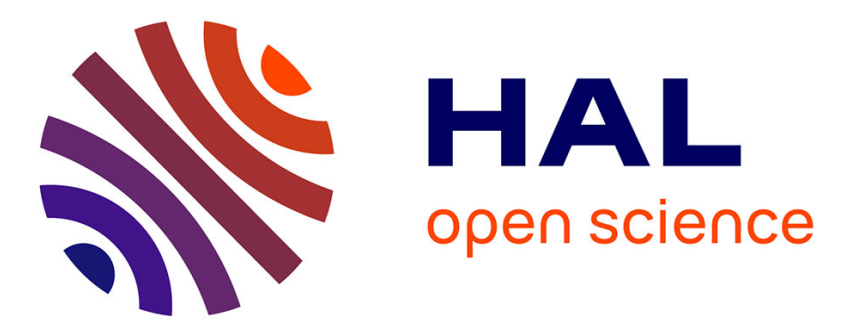

\title{
Controllability analysis and motors failures symmetry in a coaxial octorotor
}

Majd Saied, Hassan Shraim, Clovis Francis, Isabelle Fantoni, Benjamin Lussier

\section{To cite this version:}

Majd Saied, Hassan Shraim, Clovis Francis, Isabelle Fantoni, Benjamin Lussier. Controllability analysis and motors failures symmetry in a coaxial octorotor. Third International Conference on Technological Advances in Electrical, Electronics and Computer Engineering (TAEECE 2015), Apr 2015, Beirut, Lebanon. pp.245-250, 10.1109/TAEECE.2015.7113634 . hal-01215432

\section{HAL Id: hal-01215432 \\ https://hal.science/hal-01215432}

Submitted on 14 Oct 2015

HAL is a multi-disciplinary open access archive for the deposit and dissemination of scientific research documents, whether they are published or not. The documents may come from teaching and research institutions in France or abroad, or from public or private research centers.
L'archive ouverte pluridisciplinaire HAL, est destinée au dépôt et à la diffusion de documents scientifiques de niveau recherche, publiés ou non, émanant des établissements d'enseignement et de recherche français ou étrangers, des laboratoires publics ou privés. 


\title{
Controllability Analysis and Motors Failures Symmetry in a Coaxial Octorotor
}

\author{
Majd Saied ${ }^{1,2}$, Hassan Shraim ${ }^{1}$, Clovis Francis ${ }^{1}$, Isabelle Fantoni ${ }^{2}$ and Benjamin Lussier ${ }^{2}$
}

\begin{abstract}
This paper presents a fault tolerant control strategy for a coaxial octorotor regarding one motor failure. A controllability study, based on the construction of the attainable control set method, is presented for a coaxial octorotor with one or more failing motors. The fault is diagnosed using a non linear Super-Twisting sliding mode observer. The octorotor is recovered after a motor failure by controlling its dual motor. The effectiveness of this approach is illustrated by numerical simulations on an octorotor simulator.
\end{abstract}

\section{INTRODUCTION}

Fault Tolerant Control (FTC) in autonomous systems is a challenging task, especially for safety critical systems such as aircrafts. The main objective of FTC is to maintain desirable performances of the system despite faults and prevent local errors from developing into failures. In the literature, FTC approaches have been classified into two types [1]: passive and active approaches. Passive FTC is usually based on robust control and handles faults and failures without requiring information from a Fault Detection and Identification (FDI) scheme. Active FTC needs information about the faults that occur, and can be divided into projection type FTC and online reconfiguration/adaptation [2].

For quadrotors vehicles, different FTC methods were exploited to handle partial and complete actuator failures. For partial failures, in [3], the authors developed a FTC strategy combined with trajectory replanning. This approach changes the reference trajectory in function of the remaining resources in the system after a partial fault in one of the actuators. Control Re-Allocation for a modified quadrotor is proposed as a solution in [4]. Robust controllers such as sliding mode [5] were also investigated for the FTC problem for quadrotors. However, few works address the problem of FTC of quadrotor in case of total motor failure. [8] presented periodic solutions to control the quadrotor after losing one, two opposing or three propellers. The strategy consists of spinning the vehicle around an axis and then control this axis for translational movements. In [7], the quadrotor is modeled as a birotor with fixed propellers and follows a planned emergency landing trajectory when a motor failure occurs. A survey on fault tolerant control strategies for a quadrotor is given in [9]. One major drawback of quadrotors is that there is no effector redundancy, and

\footnotetext{
${ }^{1}$ Université Libanaise, Faculté de Génie, Centre de Recherche Scientifique en Ingénierie (CRSI), Liban, Email: \{cfrancis, hassan.shraim\}@ul.edu.lb

${ }^{2}$ Sorbonne Universités, Université de Technologie de Compiègne, CNRS, UMR 7253 Heudiasyc, 60200 Compiègne, France, Email: \{majd.saied, benjamin.lussier, isabelle.fantoni\}@hds.utc.fr
}

a total motor failure thus leads to a system that it is not fully controllable. Multirotors with more than four rotors (hexarotors, octorotors) have been proposed as a solution, but few works in the literature addressed the problem of FTC for multirotors with actuator redundancy. The commonly used method is the control allocation. For a star-shaped octorotor, a cascade inverse method of control allocation is proposed in [10] to allocate, after several motors failures, the controller commands to the actuators while ensuring that actuator saturation does not occur. For the same type of vehicle, [11] presented a LPV based sliding mode fault tolerant controller, and [12] presented an active FTC scheme based on Dynamic Control Allocation method. For FTC of an hexarotor, a control allocation problem formulated as a parametric program is proposed and validated experimentally in [13].

In our paper, a fault tolerant control strategy for a coaxial octorotor in case of one motor failure is presented. The fault is diagnosed using a super twisting sliding mode observer, and the system recovery is based on the built-in hardware redundancy of the octorotor.

This paper is organized as follows: Section II presents the equations governing the coaxial octorotor dynamics. Section III is dedicated to the control algorithm and section IV to the controllability study. The fault tolerant architecture is presented in section V. The results are then validated in numerical simulations on an octorotor simulator in section VI, and the paper concludes with perspectives in section VII.

\section{EQUATIONS OF MOTION}

The configuration of the coaxial octorotor is presented in Fig. 1. It is similar to a quadrotor with two coaxial counterrotating motors at the ends of each arm. Even though two coaxial rotors are less efficient than two isolated identical rotors (Fig. 2), the coaxial octorotor presents several advantages compared to the the classical star-shaped one: the classical octorotor needs more arms, and these arms need to be longer to guarantee adequate spacing among the rotors.

The dynamics of this vehicle are developed using the Euler-Lagrange approach. For simplification purpose, it is assumed that [14]:

- The structure of the vehicle is supposed rigid and symmetrical;

- The thrust and the drag are proportional to the square of the rotors speed;

- The center of gravity and the body fixed frame origin are assumed to coincide;

- The inertia matrix off-diagonal terms are zero; 


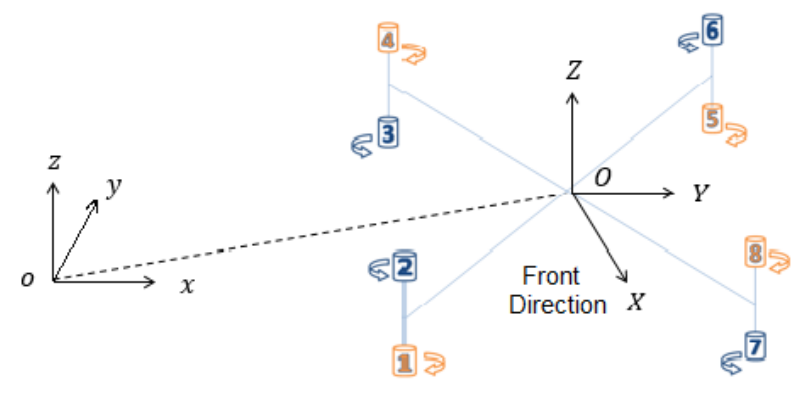

Fig. 1: The coaxial octorotor and the reference frames

- The motor dynamics are ignored.

Consider a body-fixed frame $R_{B}:\{O, X, Y, Z\}$ with the three axes originating at the center of mass of the vehicle, and an inertial frame $R_{I}:\{o, x, y, z\}$ fixed to the Earth. The rotation matrix $R$ defines the attitude of $R_{B}$ with respect to $R_{I}$ :

$R=\left[\begin{array}{ccr}C_{\theta} C_{\psi} & C_{\psi} S_{\theta} S_{\phi}-C_{\phi} S_{\psi} & C_{\phi} C_{\psi} S_{\theta}+S_{\phi} S_{\psi} \\ C_{\theta} S_{\psi} & S_{\theta} S_{\phi} S_{\psi}+C_{\phi} C_{\psi} & C_{\phi} S_{\theta} S_{\psi}-C_{\psi} S_{\phi} \\ -S_{\theta} & C_{\theta} S_{\phi} & C_{\theta} C_{\phi}\end{array}\right]$

where $\phi, \theta$ and $\psi$ are the Euler angles, and $S_{\phi, \theta, \psi}$ and $C_{\phi, \theta, \psi}$ are the sine and the cosine of the corresponding angle. The octorotor mathematical model is given as follows:

$$
\dot{X}(t)=f(X(t))+g(X(t)) \tau(t)
$$

where

$$
f(t, X)=\left[\begin{array}{c}
\dot{x} \\
0 \\
\dot{y} \\
0 \\
\dot{z} \\
-g \\
a_{\phi} \\
c_{1} q r-c_{2} q \Omega_{r} \\
a_{\theta} \\
c_{4} p r+c_{5} p \Omega_{r} \\
a_{\psi} \\
c_{7} p q
\end{array}\right], g(t, X)=\left[\begin{array}{cccc}
0 & 0 & 0 & 0 \\
b_{x} & 0 & 0 & 0 \\
0 & 0 & 0 & 0 \\
b_{y} & 0 & 0 & 0 \\
0 & 0 & 0 & 0 \\
b_{z} & 0 & 0 & 0 \\
0 & 0 & 0 & 0 \\
0 & c_{3} & 0 & 0 \\
0 & 0 & 0 & 0 \\
0 & 0 & c_{6} & 0 \\
0 & 0 & 0 & 0 \\
0 & 0 & 0 & c_{8}
\end{array}\right]
$$

The terms in (3) are defined as:

$$
\begin{aligned}
& a_{\phi}=p+q \sin (\phi) \tan (\theta)+r \cos (\phi) \tan (\theta) \\
& a_{\theta}=q \cos (\phi)-r \sin (\theta) \\
& a_{\psi}=q \sin (\phi) \sec (\theta)+r \cos (\phi) \sec (\theta) \\
& b_{x}=(\cos (\phi) \sin (\theta) \cos (\psi)+\sin (\phi) \sin (\psi)) / m \\
& b_{y}=(\cos (\phi) \sin (\theta) \sin (\psi)-\sin (\phi) \cos (\psi)) / m \\
& b_{z}=(\cos (\phi) \cos (\theta)) / m \\
& c_{1}=\left(I_{y y}-I_{z z}\right) / I_{x x}, c_{2}=J_{r} / I_{x x}, c_{3}=1 / I_{x x} \\
& c_{4}=\left(I_{z z}-I_{x x}\right) / I_{y y}, c_{5}=J_{r} / I_{y y}, c_{6}=1 / I_{y y} \\
& c_{7}=\left(I_{x x}-I_{y y}\right) / I_{z z}, c_{8}=1 / I_{z z}
\end{aligned}
$$

$\left[\begin{array}{lll}x & y & z\end{array}\right]^{T}$ and $\left[\begin{array}{lll}\phi & \theta & \psi\end{array}\right]^{T}$ denote respectively the absolute position and the Euler angles of the aerial vehicle expressed in the fixed frame $R_{I}$. $\left[\begin{array}{ll}p & q\end{array}\right]^{T}$ denote its angular velocity

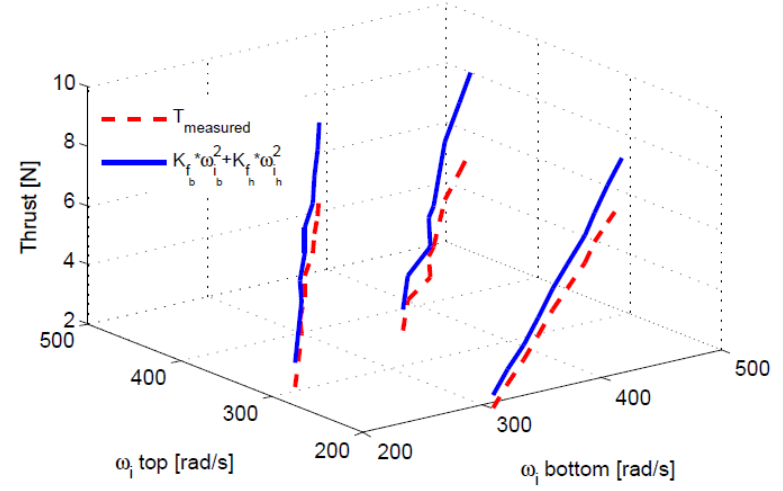

Fig. 2: Comparison between theoretical and experimental thrust of a pair of coaxial motors. The thrust is measured using a 6 degree of freedom force/torque sensor.

in the body frame $R_{B} ; m$ is the mass of the aerial vehicle; $I_{x x}, I_{y y}, I_{z z}$ represent the inertia of the octorotor about the three axis; $J_{r}$ is the rotor inertia.

The rotating speed relation between the body coordinates and the generalized coordinates can be written as follows:

$$
\left[\begin{array}{c}
\dot{\phi} \\
\dot{\theta} \\
\dot{\psi}
\end{array}\right]=\left[\begin{array}{ccc}
1 & \sin \phi \tan \theta & \cos \phi \tan \theta \\
0 & \cos \phi & -\sin \phi \\
0 & \sin \phi / \cos \theta & \cos \phi / \cos \theta
\end{array}\right]\left[\begin{array}{l}
p \\
q \\
r
\end{array}\right]
$$

In case of small angles, this matrix is identical to the identity matrix $I_{3}$, and then the following approximations will be verified: $\dot{\phi}=p, \dot{\theta}=q$ and $\dot{\psi}=r$.

The inputs of the system are:

$$
\tau(t)=\left[u_{f} \tau_{\phi} \tau_{\theta} \tau_{\psi}\right]^{T}
$$

representing the total thrust, roll torque, pitch torque and yaw torque respectively. The variable

$$
\Omega_{r}=\omega_{2}+\omega_{3}+\omega_{6}+\omega_{7}-\omega_{1}-\omega_{4}-\omega_{5}-\omega_{8}
$$

is the overall residual propeller speed from the unbalanced rotor rotation, and $\omega_{1} \ldots \omega_{8}$ are the motors speeds. The input torque and force can be related to the squared speeds $\omega_{i}^{2}$ through:

$$
\begin{aligned}
& u_{f}=F_{12}+F_{34}+F_{56}+F_{78} \\
& \tau_{\phi}=\left(F_{78}+F_{56}-F_{34}-F_{12}\right) * l * \sqrt{2} / 2 \\
& \tau_{\theta}=\left(F_{34}+F_{56}-F_{78}-F_{12}\right) * l * \sqrt{2} / 2 \\
& \tau_{\psi}=\left(\tau_{2}+\tau_{3}+\tau_{6}+\tau_{7}\right)-\left(\tau_{1}+\tau_{4}+\tau_{5}+\tau_{8}\right)
\end{aligned}
$$

The thrust produced by each pair of coaxial rotors $i$ and $j$ is given by [15] :

$$
\begin{gathered}
F_{i j}=\alpha_{i j} *\left(F_{i}+F_{j}\right) *\left(1+\frac{S_{s}}{S_{\text {prop }}}\right) \\
F_{i}=K_{f} * \omega_{i}^{2}
\end{gathered}
$$

$\alpha_{i j}$ is the coefficient of loss of aerodynamic efficiency due to the aerodynamic interference between the upper and lower rotors of each pair of coaxial rotors. $S=\left(1+\frac{S_{s}}{S_{\text {prop }}}\right)$ represents the shape factor of the propellers, with $S_{s}$ denoting the propeller's surface and $S_{\text {prop }}$ the surface of the circle that 
the propeller would make when rotating. $K_{f}$ is the thrust factor. The torque produced by each rotor is expressed by:

$$
\tau_{i}=K_{t} * \omega_{i}^{2}
$$

with $K_{t}$ is the reaction torque coefficient.

\section{Control StRATEGy}

A control strategy for stabilizing the octorotor while hovering is presented in this section. The altitude and the yaw positions are controlled by a PID controller that makes use of information obtained respectively from an ultrasonic sensor and an Inertial Measurement Unit (IMU):

$$
u=K_{p} e+K_{d} \dot{e}+K_{I} \int_{0}^{t} e(\tau) d \tau
$$

$K_{p}, K_{d}$ and $K_{I}$ are the controller's gains, and $e$ is the state error compared to the desired position.

The roll and pitch angles are controlled using saturation functions, where each state is bounded separately (12). The stability of this control law is proved in [17].

$$
\begin{gathered}
\tau_{\phi}=\frac{I_{x x}}{g}\left[\sigma_{p y}\left(k_{p y}\left(y-y_{d}\right)\right)+\sigma_{d y}\left(k_{d y} \dot{y}\right)-\right. \\
\left.\sigma_{p \phi}\left(k_{p \phi} \phi\right)-\sigma_{d \phi}\left(k_{d \phi} \dot{\phi}\right)\right] \\
\tau_{\theta}=-\frac{I_{y y}}{g}\left[\sigma_{p x}\left(k_{p x}\left(x-x_{d}\right)\right)+\sigma_{d x}\left(k_{d x} \dot{x}\right)-\right. \\
\left.\sigma_{p \theta}\left(k_{p \theta} \theta\right)-\sigma_{d \theta}\left(k_{d \theta} \dot{\theta}\right)\right]
\end{gathered}
$$

where $k_{p y}, k_{d y}, k_{p \phi}, k_{d \phi}, k_{p x}, k_{d x}, k_{p \theta}$ and $k_{d \theta}$ are positive gains, and $\sigma_{p y}, \sigma_{d y}, \sigma_{p \phi}, \sigma_{d \phi}, \sigma_{p x}, \sigma_{d x}, \sigma_{p \theta}$, and $\sigma_{d \theta}$ are saturation functions defined as follows:

$$
\begin{cases}\sigma_{b_{i}}(s)=b_{i} & \text { if } \quad s>b_{i} \\ \sigma_{b_{i}}(s)=s & \text { if } \quad-b_{i}<s<b_{i} \\ \sigma_{b_{i}}(s)=-b_{i} & \text { if } \quad s<-b_{i} \\ \text { IV. CONTROLLABILITY } & \text { CONTR }\end{cases}
$$

\section{Controllability}

Before establishing a fault tolerant control strategy, the controllability of the octorotor in case of motors failures is studied in this section. The control inputs of the multirotors are constrained to be non negative (the rotors can only provide unidirectional lift, upward or downward). This makes the classical controllability theories not sufficient to test the controllability of the octorotor.

Static controllability of the octorotor is investigated below using the method based on the construction of the attainable control set (ACS) developed in [13]. The ACS is a subspace in TRPY (Thrust Force; Roll, Pitch, Yaw torques) which defines the limits in thrust and torque that can be allocated when the motors speeds constraints are satisfied. It is obtained by mapping the boundary of the motors constraints set to a virtual control set by using the control effectiveness matrix B:

$$
B=\left[\begin{array}{ccc}
t_{1} & \ldots & t_{8} \\
r_{1} & \ldots & r_{8} \\
p_{1} & \ldots & p_{8} \\
y_{1} & \cdots & y_{8}
\end{array}\right]
$$

with:

$$
\begin{aligned}
& t_{i}=\sigma_{i j} \cdot K_{f} \cdot S \\
& r_{i}=d_{r_{i}} \cdot \sigma_{i j} \cdot K_{f} \cdot S \cdot l \cdot \sqrt{2} / 2 \\
& p_{i}=d_{p_{i}} \cdot \sigma_{i j} \cdot K_{f} \cdot S \cdot l \cdot \sqrt{2} / 2 \\
& y_{i}=d_{y_{i}} \cdot K_{t}
\end{aligned}
$$

$t_{i}, r_{i}, p_{i}$ and $y_{i}$ are respectively the thrust and the torques around the three axes produced by the actuator $i . d_{r_{i} / p_{i} / y_{i}}$ is either 1 or -1 depending on whether the force created by the motor generates a positive or negative moment (determined by $(8))$.

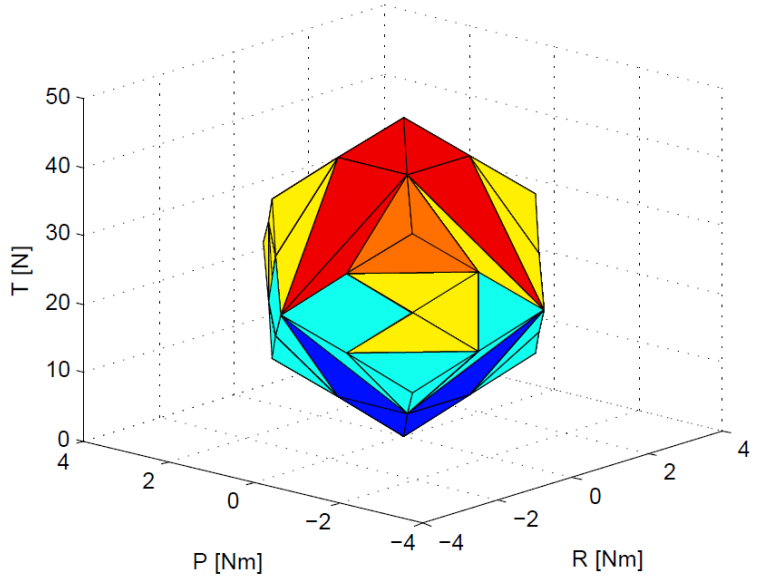

Fig. 3: Attainable control set for a coaxial octorotor in fault-free case cut at Y=0; (T: Thrust, R: Roll torque, P: Pitch torque, Y: Yaw torque).

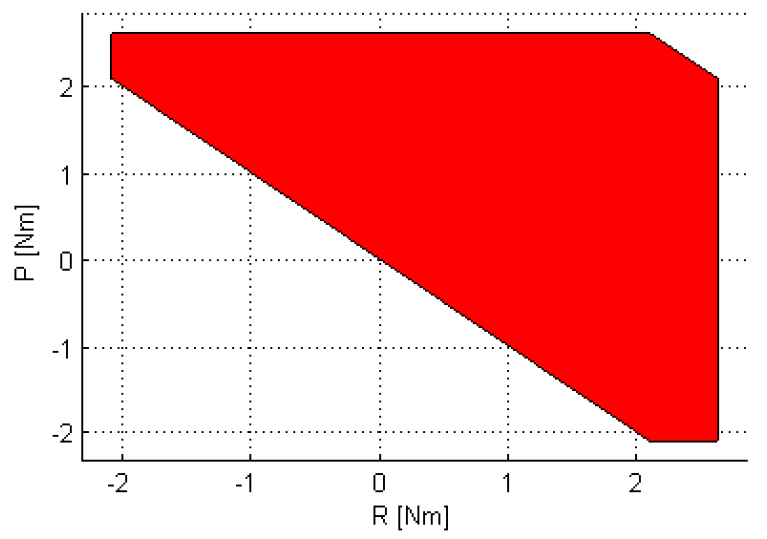

Fig. 4: Attainable control set for a coaxial octorotor, in case of failures of motors 1 and 2 , cut at $\mathrm{Y}=0$ and $\mathrm{T}=\mathrm{mg}$; ( $\mathrm{T}$ : Thrust, R: Roll torque, $\mathrm{P}$ : Pitch torque, Y: Yaw torque).

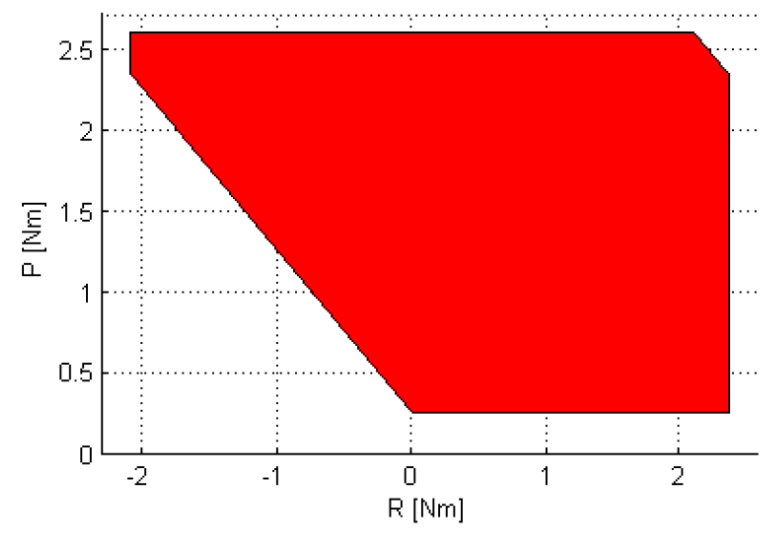

Fig. 5: Attainable control set for a coaxial octorotor, in case of failures of motors 1, 2 and 3, cut at $\mathrm{Y}=0$ and $\mathrm{T}=\mathrm{mg}$; (T: Thrust, R: Roll torque, P: Pitch torque, Y: Yaw torque). 
According to [13], to assess the controllability of the octorotor, the 4-dimensional polytope $T R P Y$ (Fig. 3) is cut at the nominal flying conditions $T=m g$ and $Y=0$. If the origin of the $R-P$ plane is contained in the polygon, then the octorotor is controllable.

For a coaxial octorotor, there are 162 fault combinations considering that at least four motors are healthy. The controllability study shows that a coaxial octorotor is not controllable in case of failures of two coaxial motors as shown in Fig. 4 and Fig. 5. In these cases, reduced attitude controllability should be investigated. Moreover, we see in Fig. 4 and 5 that, as the point $(\mathrm{R}=0, \mathrm{P}=0, \mathrm{Y}=0)$ is not in the attainable control set of the system (it is at this set's limit in Fig. 4, and outside in Fig. 5), the system is unable to hover as soon as two coaxial motors fail.

\section{Fault Tolerant Control Strategy}

Active FTC requires information about the fault, its location and its severity to apply the corresponding system recovery law. This is done via an error detection and fault diagnosis module.

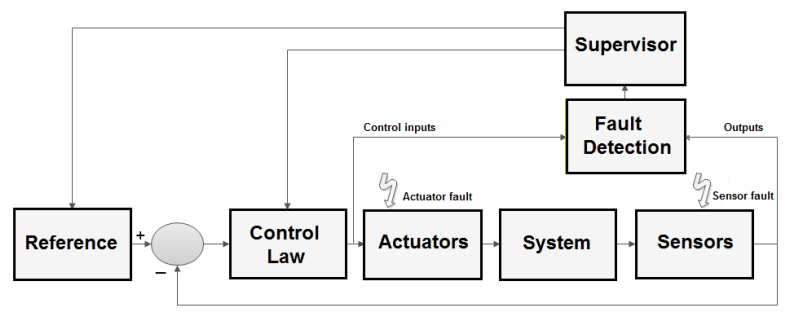

Fig. 6: Control system using error detection and supervisor modules

The octorotor presents redundancy in actuators, and thus identifying the failed motor can be a challenging task. Most of the researches in FTC of multirotors with redundant actuators do not consider Fault Diagnosis in their study assuming that the faults, their locations and magnitudes are already known. In our system, a second order sliding mode based on the super-Twisting algorithm is used for error detection and fault diagnosis in the octorotor. This strategy is proposed in [16]. The main idea is to use the equivalent output injection to estimate the unknown input and thus to identify losses in the actuators.

After the identification of the failed motor, a recovery strategy is applied, based on the built-in hardware redundancy of the octorotor and a mathematical analysis of the octorotor dynamics (8). It consists of synchronising the failed motor and its dual one to compensate the losses in roll, pitch and yaw torques. A reconfiguration of the control law is necessary to compensate the altitude loss. The duality between motors is deduced from their geometrical distribution (Table I).

In normal flight, the speed of a motor $i$ is given by:

$$
\omega_{i}=\sqrt{\frac{1}{8} *\left(\frac{u_{f}}{\alpha_{i j} S K_{f}} \pm \frac{\tau_{\phi}}{\alpha_{i j} S K_{f} d} \pm \frac{\tau_{\theta}}{\alpha_{i j} S K_{f} d} \pm \frac{\tau_{\psi}}{K_{t}}\right)}
$$

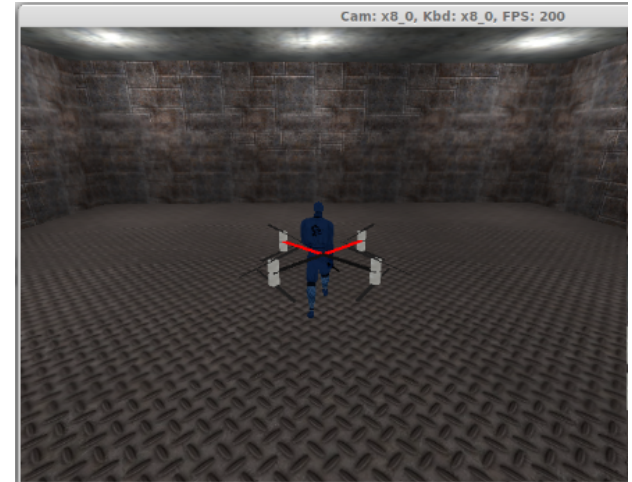

Fig. 7: Simulator of the octorotor

After diagnosing the failure, a correction factor is added to ensure that the same total thrust and moments as in normal flight are generated, resulting in equation (17):

$\omega_{i}=p_{i} \sqrt{\left(\frac{1}{8}+c_{i}\right)\left(\frac{u_{f}}{\alpha_{i j} S K_{f}} \pm \frac{\tau_{\phi}}{\alpha_{i j} S K_{f} d} \pm \frac{\tau_{\theta}}{\alpha_{i j} S K_{f} d} \pm \frac{\tau_{\psi}}{K_{t}}\right)}$

where $p_{i}$ is the percentage of motor failure and $c_{i}$ is the correction factor.

\begin{tabular}{cc}
\hline Motor & Dual Motor \\
\hline \hline 1 & 6 \\
\hline 2 & 5 \\
\hline 3 & 8 \\
\hline 4 & 7 \\
\hline
\end{tabular}

TABLE I: Duality between motors

\section{VALIDATION}

In order to test the performance of the FTC strategy, simulations were carried out using an octorotor simulator (Fig. 7). Before validating results in real experiments, all the algorithms are first tested on a simulator written in $\mathrm{C}++$ developed at Heudiasyc laboratory. Its purpose is to execute on a computer the same code used in the real UAV. To do this, the computer is running Linux with its real-time extension Xenomai. The UAV evolves in a 3D environment thanks to the Irrlicht $3 \mathrm{D}$ engine. The UAV program is connected to a ground station from which parameters (control laws, filters...) can be changed during the flight. This approach allows us a significant time savings as well as safely injecting faults in the system. A real experiment is presented in [18].

The model's parameters are given in Table II. The actuator faults are simulated. The octorotor is brought to a hovering stable flight, then the motor 6 is turned off at time $\mathrm{t}=36.2 \mathrm{~s}$ and after $0.44 \mathrm{~s}$ (time needed by the diagnosis module to detect the error and identify the failing motor), its dual motor (motor 1) is turned off to compensate the loss. 


\begin{tabular}{|c|c|c|}
\hline$K_{f}$ & Thrust factor & $3 * 10^{-5} \mathrm{Ns}^{2} / \mathrm{rad}^{2}$ \\
\hline$K_{t}$ & drag factor & $7 * 10^{-7} \mathrm{Nm} / \mathrm{rad}^{2}$ \\
\hline $\mathrm{m}$ & mass of the vehicle & $1.6 \mathrm{~kg}$ \\
\hline $\mathrm{l}$ & length of the arm & $0.23 \mathrm{~m}$ \\
\hline$I_{x x}, I_{y y}$ & Inertia & $4.2 * 10^{-2} \mathrm{Kg} \cdot \mathrm{m}^{2}$ \\
\hline$I_{z z}$ & Inertia & $7.5 * 10^{-2} \mathrm{Kg} \cdot \mathrm{m}^{2}$ \\
\hline
\end{tabular}

TABLE II: The model's parameters

In Fig. 9, the three Euler angles are shown. The vertical dashed lines indicate the fault injection time. It can be observed that the angles deviate quickly from the stable position after the failure has occurred with the recovery not being applied yet. However, they return to their initial values when the recovery is performed after a delay of $0.44 \mathrm{~s}$. Fig. 8 shows the altitude $z$ after a fault occurrence.

To demonstrate the motors failures symmetry, a second scenario is presented where a failure is injected on motor 3 . Fig. 10 and Fig. 11 reveals that the behavior is similar to a failure on motor 6 (with corresponding sign changes in the Euler angles). Further experiments on other motors showed again the symmetry considering the failed motor.

The main advantage of this method is its deterministic character and fast computation in comparison with other methods which need the resolution of optimization problems online. Theoretically, it is able to recover the system after one motor failure even if the octorotor is not in hovering flight. However, its main drawback is that it will not be able to recover the system after some two or more motors failures cases.

The influence of the detection time is investigated in Fig. 12 and Fig. 13. It can be seen that the smaller the detection time, the smaller the deviation of the octorotor from the equilibrium position. The system is then restored faster. The configuration delay is a critical but somewhat lax parameter since it is possible to restore the system after a delay greater than $1 \mathrm{~s}$.

\section{CONCLUSION}

This paper presents a FTC method for a coaxial octorotor regarding one motor failure. Particularly, it presents a controllability study on a coaxial octorotor, and the motors failures symmetry of our recovery architecture. In future works, we intend to consider successive motors failures, to further recover the octorotor from critical cases such as when two coaxial motors fails, and to compare experimentally this strategy with FTC based on control allocation and robust controller.

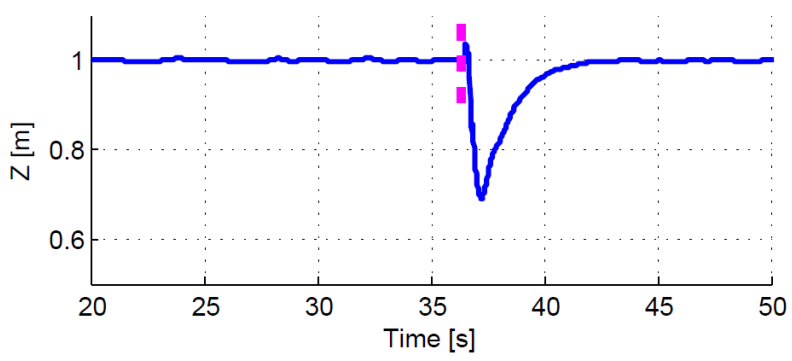

Fig. 8: Altitude (m] after fault injection at time $\mathrm{t}=36.2 \mathrm{~s}$ on motor 6 .
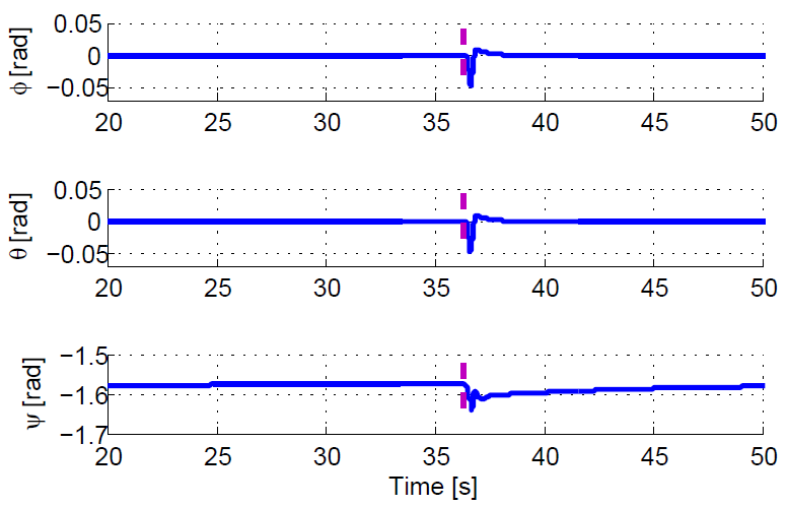

Fig. 9: Euler angles $[\mathrm{rad}]$ after fault injection at time $\mathrm{t}=36.2 \mathrm{~s}$ on motor 6 .

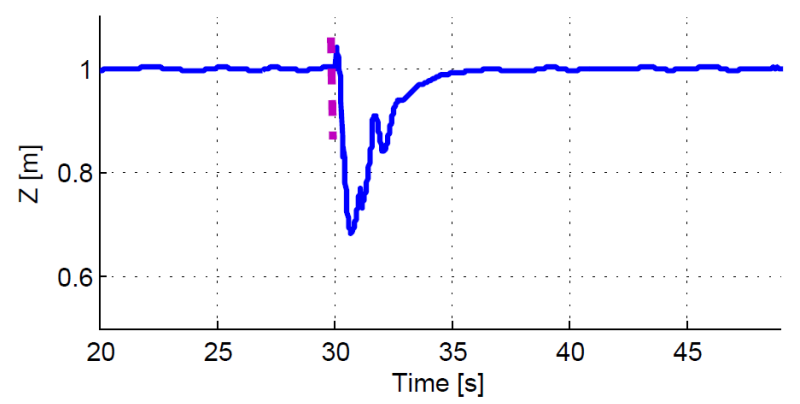

Fig. 10: Altitude [m] after fault injection at time $\mathrm{t}=30 \mathrm{~s}$ on motor 3 .
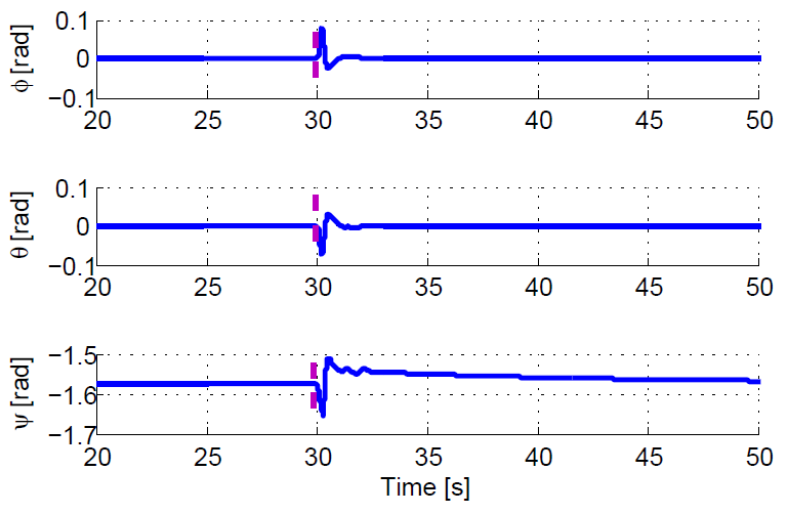

Fig. 11: Euler angles [rad] after fault injection at time $t=30$ s on motor 3 .

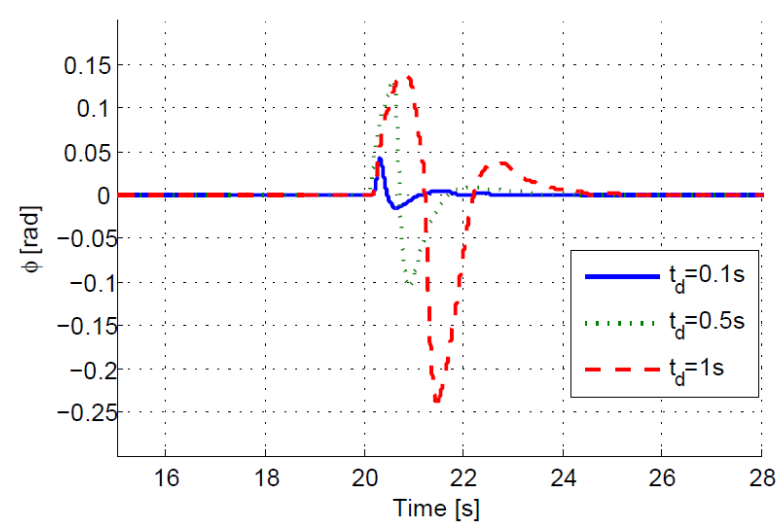

Fig. 12: Euler angles [rad] after fault injection at time $t=20$ s on motor 3 with different configuration delays. 


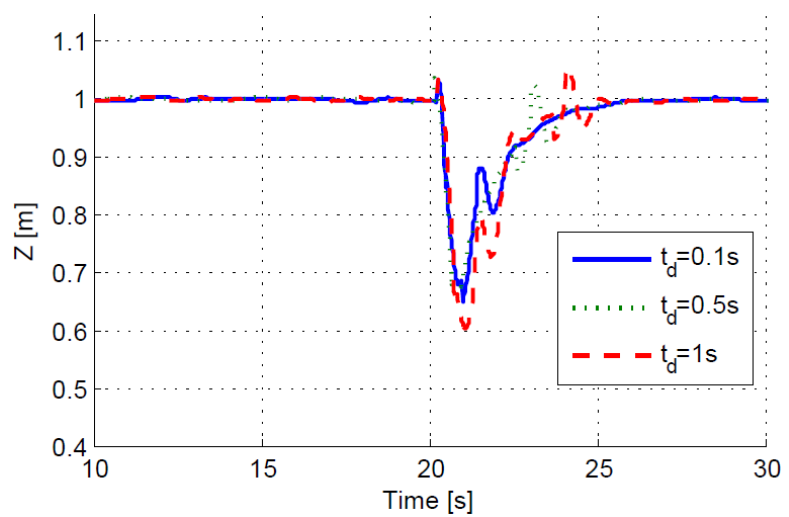

Fig. 13: Altitude $[\mathrm{m}]$ after fault injection at time $\mathrm{t}=20 \mathrm{~s}$ on motor 3 with different configuration delays.

\section{ACKNOWLEDGEMENTS}

This work was carried out and funded in the framework of the Labex MS2T (Reference ANR-11-IDEX-0004-02) and the ROBOTEX Equipment of Excellence (Reference ANR-10- EQPX-44). They were supported by the French Government, through the program Investments for the future managed by the National Agency for Research.

The authors would like to thank the Lebanese University research support program.

\section{REFERENCES}

[1] Y. Zhang and J. Jiang, "Bibliographical review on reconfigurable fault-tolerant control systems," IFAC Symposuim on Fault Detection, Supervision and Safety for Technical Processes, Washington, USA, June 9-11, 2003, pp. 265-276.

[2] H. Alwi, C. Edwards and C. Pin Tan, "Fault Detection and FaultTolerant Control Using Sliding Modes," Springer, 2011.

[3] A. Chamseddine, Y. Zhang, C. A. Rabbath, C. Join, and D. Theilliol, "Flatness-based trajectory planning/replanning for a quadrotor unmanned aerial vehicle," IEEE Transactions on Aerospace and Electronic Systems, vol. 48, no. 4, pp. 2832-2848, 2012.

[4] I. Sadeghzadeh, A. Chamseddine, Y.M. Zhang, and D. Theilliol, "Control Allocation and Re-allocation for a Modified Quadrotor Helicopter against Actuator Faults," Proc. of the 8th IFAC Symposium on Fault Detection, Supervision and Safety for Technical Processes, Mexico City, Mexico, August, 2012, pp. 247-252.

[5] T. Li, Y. Zhang and B. W. Gordon, "Nonlinear Fault-Tolerant Control of a Quadrotor UAV Based on Sliding Mode Control Technique," IFAC Symposium on Fault Detection, Supervision and Safety of Technical Processes, Mexico City, Mexico, August 29-31, 2012, pp. 1317-1322.

[6] A. Freddi, A. Lanzon, and S. Longhi, "A feedback linearization approach to fault tolerance in quadrotor vehicles," IFAC World Congress, Milano, Italy, 2011, pp. 5413-5418.

[7] V. Lippiello, F. Ruggiero and D. Serra, "Emergency Landing for a Quadrotor in Case of a Propeller Failure: A Backstepping Approach," International Conference on Intelligent Robots and Systems (IROS), Chicago, IL, USA, September 14-18, 2014, pp. 4782-4788.

[8] M.W. Mueller and R. D' Andrea, "Stability and control of a quadrocopter despite the complete loss of one, two, or three propellers," IEEE International Conference on Robotics and Automation, Hong Kong, China, May 31-June 7, 2014, pp. 45-52.

[9] Y. Zhang, A. Chamseddine, C. Rabbath, B. Gordon, C.-Y. Su, S. Rakheja, C. Fulford, J. Apkarian, and P. Gosselin, "Development of advanced FDD and FTC techniques with application to an unmanned quadrotor helicopter testbed," Journal of the Franklin Institute, vol. 350, no. 9, pp. 2396-2422, 2013.

[10] A. Marks, J. F. Whidborne, and I. Yamamoto, "Control allocation for fault tolerant control of a VTOL octorotor," UKACC International Conference on Control, Cardiff, Sept. 3-5, 2012, pp. 357-362.
[11] H. Alwi and C. Edwards, "Fault Tolerant Control of an Octorotor Using LPV based Sliding Mode Control Allocation," American Control Conference (ACC), Washington, DC, USA, June 17-19, 2013, pp. 65056510.

[12] A. Merheb, H. Nourra and F. Bateman, "Active Fault Tolerant Control of Octorotor UAV using Dynamic Control Allocation," International Conference on Intelligent Unmanned Systems, Montreal, Quebec, Canada, Sept. 29-Oct. 1, 2014.

[13] T. Schneider, G. Ducard, K. Rudin and P. Strupler, "Fault-tolerant Control Allocation for Multirotor Helicopters Using Parametric Programming," International Micro Air Vehicle Conference and Flight Competition, Braunschweig, Germany, July, 2012.

[14] S. Bouabdallah, "Design and control of quadrotors with applications to autonomous flying," $\mathrm{PhD}$ thesis, Ecole Polytechnique Fédérale De Lausanne, 2007.

[15] A. Koehl, "Modélisation, Observation et Commande d'un drone miniature birotor coaxial," Ph.D thesis, University of Nancy, 2012.

[16] M. Saied, H. Shraim, C. Francis, I. Fantoni and B. Lussier, "Actuator Fault Diagnosis in an Octorotor UAV Using Sliding Modes Technique: Theory and Experimentation," To appear in Europeen Control Conference, Linz, Austria, July 15-17, 2015.

[17] G. Sanahuja, P. Castillo and A. Sanchez, "Stabilization of n integrators in cascade with bounded input with experimental application to a VTOL laboratory system," Int. Journal of Robust and Nonlinear Control, vol. 20, no. 10, pp. 1129-1139, 2010.

[18] M. Saied, B. Lussier, I. Fantoni, C. Francis, H. Shraim and G. Sanahuja, "Fault Diagnosis and Fault-Tolerant Control Strategy for Rotor Failure in an Octorotor," To appear in International Conference on Robotics and Automation (ICRA), Washington, May 26-30, 2015. 